\title{
ON BOOLEAN ALGEBRAS OF PROJECTIONS
}

\author{
by J. A. ERDOS \\ (Received 12 August, 1975)
}

In spectral theory on Banach spaces, certain more incisive results hold when the underlying space is weakly complete (that is, weakly sequentially complete). The standard proofs rely on the following deep theorem: any bounded linear map from the algebra of all complex continuous functions on a compact Hausdorff space to a weakly complete Banach space is weakly compact. The proof of this result depends in turn on a considerable amount of measure-theoretic machinery (see [4, Section VI.7]). We present here some alternative methods which avoid these technicalities. The results are then used to give an example of a set of projections, each having unit norm, which generate an unbounded Boolean algebra.

The results in question are as follows. Standard terminology (see, for example [4]) will be used.

THEOREM 1. A strongly closed bounded Boolean algebra of projections in a weakly complete Banach space is complete.

Proof. It is true in general ([4, Lemma XVIII.3.4]) that a strongly closed Boolean algebra $\mathscr{B}$ is complete if every increasing net of elements of $\mathscr{B}$ is strongly convergent.

Suppose now that $\mathscr{B}$ is a Boolean algebra of projections on the weakly complete space $X$ and that $\|P\| \leqq K$, for all $P \in \mathscr{B}$. Let $\left\{P_{\alpha}\right\}$ be an increasing net of elements of $\mathscr{B}$. If $\left\{P_{\alpha}\right\}$ is not strongly convergent then for some $x \in X,\left\{P_{\alpha} x\right\}$ is not norm convergent and so is not a Cauchy net in $(X,\|\cdot\|)$. Hence, for some $k>0$, we can find an increasing sequence $\left\{\alpha_{i}\right\}$ such that, (writing $P_{i}$ for $P_{\alpha_{i}}$ ),

$$
\left\|\left(P_{i+1}-P_{i}\right) x\right\|>k \text {. }
$$

We shall in due course obtain a contradiction to this statement.

First we show that $\left\{P_{i} x\right\}$ is a weak Cauchy sequence. Since every element of the dual $X^{*}$ of $X$ is a linear combination of two real-valued elements of $X^{*}$, it is sufficient to show that $\left\{\psi\left(P_{i} x\right)\right\}$ is a Cauchy sequence for every real-valued element $\psi$ of $X^{*}$. Let

$$
\mathscr{I}^{+}=\left\{i: \psi\left[\left(P_{i+1}-P_{i}\right) x\right] \geqq 0\right\} \text { and } \mathscr{I}^{-}=\left\{i: \psi\left[\left(P_{i+1}-P_{i}\right) x\right]<0\right\} .
$$

Then for every integer $n$,

$$
\begin{aligned}
\sum_{i=1}^{n}\left|\psi\left[\left(P_{i+1}-P_{i}\right) x\right]\right| & =\sum_{i \in \mathscr{S}+, i \leqq n} \psi\left[\left(P_{i+1}-P_{i}\right) x\right]-\sum_{i \in \mathcal{S}-, i \leqq n} \psi\left[\left(P_{i+1}-P_{i}\right) x\right] \\
& =\psi\left[\sum_{i \in \mathcal{S}} \sum_{i \leqq n}\left(P_{i+1}-P_{i}\right) x\right]-\psi\left[\sum_{i \in \mathcal{S}} \sum_{, i \leqq n}\left(P_{i+1}-P_{i}\right) x\right] \\
& \leqq 2 K\|\psi\|\|x\| .
\end{aligned}
$$

Hence $\sum \psi\left[\left(P_{i+1}-P_{i}\right) x\right]$ is convergent and so $\left\{\psi\left(P_{i} x\right)\right\}$ is a convergent sequence. Since $X$ is weakly complete, it follows that $\left\{P_{i} x\right\}$ converges weakly to some element $y$ of $X$.

Glasgow Math. J. 18 (1977) 69-72. 
For any $\phi \in X^{*}$ and any integers $i$ and $j$ with $i>j$, since $P_{i}>P_{j}$ we have

$$
\left|\phi\left[P_{j}(x-y)\right]\right|=\left|\phi\left(P_{j} P_{i} x-P_{j} y\right)\right|=\left|P_{j}^{*} \phi\left(P_{i} x-y\right)\right| .
$$

The limit, as $i$ tends to infinity, of the right-hand term is zero and so it follows that

$$
P_{j} x=P_{j} y,
$$

for any integer $j$. Let $N_{i}$ be the range of $P_{i}$. Then $\bigcup_{i=1}^{\infty} N_{i}$ is a linear subspace of $X$ and so its norm closure is the same as its weak closure. Since $y$ is in the weak closure it follows that for any $\varepsilon>0$, there exists an integer $i$ and a vector $z \in N_{i}$ such that $\|y-z\|<\varepsilon$. Then, since $P_{i+1}>P_{i}, P_{i} z=P_{i+1} z$ and so, using (2)

Hence

$$
P_{i+1} x-P_{i} x=P_{i+1} y-P_{i} y=\left(P_{i+1}-P_{i}\right)(y-z) .
$$

$$
\left\|\left(P_{i+1}-P_{i}\right) x\right\|<K \varepsilon .
$$

Since $\varepsilon$ is arbitrary this contradicts (1) and so completes the proof.

The above proof uses ideas from Barry [2]. A shorter proof could be given by referring to the result of [2]. However, giving the proof in full serves to underline its elementary nature.

THEOREM 2. Let $f \mapsto T_{f}$ be a continuous homomorphism from the algebra $C(\Lambda)$ of all continuous complex functions on the complex Hausdorff space $\Lambda$ into the algebra of bounded linear operators on a weakly complete Banach space $X$. Then there exists a unique spectral measure $E(\cdot)$ on $X$ defined on the Baire sets and countably additive in the strong operator topology such that, for all $f \in C(\Lambda)$,

$$
T_{f}=\int_{\Lambda} f(\lambda) E(d \hat{\lambda})
$$

Proof. Without the hypothesis that $X$ is weakly complete, an argument based on the Riesz representation theorem shows that there exists a unique spectral measure $F(\cdot)$ on the dual of $X$ such that for all $f \in C(\Lambda)$

$$
T_{f}^{*}=\int_{\Lambda} f(\hat{\lambda}) F(d \hat{\lambda})
$$

(see [4, Theorem XVII.2.4]). Thus it is only required to show that for every Baire set $\delta$, there exists an operator $E(\delta)$ on $X$ such that $E(\delta)^{*}=F(\delta)$.

We first assume that $\delta$ is compact. Then there exists a decreasing sequence $\left(f_{n}\right)$ of continuous functions converging pointwise to the characteristic function of $\delta$ (see $[5, \mathrm{p} .240$, Theorem $\mathrm{A}]$ ). For any $x \in X, \phi \in X^{*}$ we have

$$
\phi\left(T_{f_{n}} x\right)=\left(T_{f_{n}}^{*} \phi\right)(x)=\int_{\Lambda} f_{n}(\lambda) F(d \lambda) \phi(x)
$$

and so, by the monotone convergence theorem, the sequence $\left\{\phi\left(T_{f_{n}} x\right)\right\}$ converges to $(F(\delta) \phi)(x)$. Since $X$ is weakly complete, this shows that $\left\{T_{f_{n}}\right\}$ converges in the weak operator topology to some operator $E(\delta)$. Clearly $E(\delta)^{*}=F(\delta)$. 
For an arbitrary Baire set $\delta$, note that the set $\{E(\beta): \beta$ compact, $\beta \subseteq \delta\}$ forms an increasing net of projections. The Boolean algebra generated by this set is bounded since for any element $E$ we have $E^{*}=F(\gamma)$ for some Baire set $\gamma$. Hence by Theorem 1 this net converges to some projection which we call $E(\delta)$. Then for all $x \in X, \phi \in X^{*}$, since $(F(\cdot) \phi)(x)$ is a regular measure,

$$
\phi(E(\delta) x)=\lim _{\beta} \phi(E(\beta) x)=\lim _{\beta}(F(\beta) \phi)(x)=(F(\delta) \phi)(x)
$$

and so $E(\delta)^{*}=F(\delta)$. This completes the proof.

The above theorem is slightly weaker than the standard result (Theorem XVIII.2.5 of [4]) in that the measure is defined on the Baire sets rather than the Borel sets. However, in most applications $\Lambda$ is metrisable and so the Baire and Borel sets coincide.

In the following example, the underlying Banach space is the trace class $\mathscr{C}_{1}$ of operators on the Hilbert space $H$. If $A \in \mathscr{C}_{1}$ then $\tau(A)$ denotes the trace of $A$. We refer to [4, Chapter XI] for properties of $\mathscr{C}_{1}$. In particular recall that every element of the dual of $\mathscr{C}_{1}$ is of the form $A \mapsto \tau(X A)$ where $X$ is some bounded linear operator on $H$. Using this fact it is easy to prove that $\mathscr{C}_{1}$ is weakly complete; alternatively the weak completeness of $\mathscr{C}_{1}$ may be deduced from Corollary III.3 of [1] since $\mathscr{C}_{1}$ is the pre-dual of a $W^{*}$-algebra.

EXAMPLE. Let $\&$ be a set of commuting self-adjoint projections on the Hilbert space $H$ which is totally ordered under the natural ordering of projections. Suppose that the commutant $\mathscr{E}^{\prime}$ of $\mathscr{E}$ contains no non-zero compact operator. (For example, on $L^{2}[0,1]$, if $E_{t}$ is the operator of multiplication by the characteristic function of $[0, t]$, then $\mathscr{E}=\left\{E_{t}: 0 \leqq t \leqq 1\right\}$ is such a set.) Let $\mathscr{D}$ be the set of all finite subsets of $\mathscr{E}$ directed by inclusion. If $\Delta=\left\{E_{1}, E_{2}\right.$, $\left.\ldots, E_{n}\right\} \in \mathscr{D}$, we define $P_{\Delta}: \mathscr{B}(H) \rightarrow \mathscr{B}(H)$ by

$$
P_{\Delta}(T)=\sum_{i=0}^{n}\left(E_{i+1}-E_{i}\right) T\left(E_{i+1}-E_{i}\right)
$$

where (for notational convenience) $E_{0}=0$ and $E_{n+1}=I$. Since the trace class is an ideal of $\mathscr{B}(H)$, if $T \in \mathscr{C}_{1}$ then $P_{\Delta}(T) \in \mathscr{C}_{1}$. We shall prove that the set $\left\{P_{\Delta}: \Delta \in \mathscr{D}\right\}$, regarded as operators on $\mathscr{C}_{1}$, form a set of projections of norm 1 but the Boolean algebra they generate is not uniformly bounded.

It is clear that $P_{\Delta}$ is a projection. Also, for all $x \in H$,

$$
\begin{aligned}
\left\|P_{\Delta}(T) x\right\|^{2} & =\sum_{i=0}^{n}\left\|\left(E_{i+1}-E_{i}\right) T\left(E_{i+1}-E_{i}\right) x\right\|^{2} \\
& \leqq\|T\|^{2} \sum_{i=0}^{n}\left\|\left(E_{i+1}-E_{i}\right) x\right\|^{2} \\
& =\|T\|^{2}\|x\|^{2},
\end{aligned}
$$

where $\|\cdot\|$ is the norm in $\mathscr{B}(H)$. Hence $\left\|P_{\Delta}(T)\right\| \leqq\|T\|$. Now if $A \in \mathscr{C}_{1}$, for all $T \in \mathscr{B}(H)$, 
since $\tau$ is linear and satisfies $\tau(X Y)=\tau(Y X)$,

$$
\begin{aligned}
\tau\left[T P_{\Delta}(A)\right] & =\sum_{i=0}^{n} \tau\left[T\left(E_{i+1}-E_{i}\right) A\left(E_{i+1}-E_{i}\right)\right] \\
& =\sum_{i=0}^{n} \tau\left[\left(E_{i+1}-E_{i}\right) T\left(E_{i+1}-E_{i}\right) A\right] \\
& =\tau\left[P_{\Delta}(T) A\right] .
\end{aligned}
$$

Therefore, denoting the norm in $\mathscr{C}_{1}$ by \|\|$^{*}$,

$$
\left\|P_{\Delta}(A)\right\|_{1}=\sup _{\|T\| \leqq 1} \tau\left[T P_{\Delta}(A)\right]=\sup _{\|T\| \leqq 1} \tau\left[P_{\Delta}(T) A\right] \leqq \sup _{\|T\| \leqq 1}\left\|P_{\Delta}(T)\right\|\|A\|_{1} \leqq\|A\|_{1}
$$

and so $P_{\Delta}$ is a projection of norm 1 on $\mathscr{C}_{1}$.

We now prove that the Boolean algebra generated by $\left\{P_{\Delta}: \Delta \in \mathscr{D}\right\}$ is not uniformly bounded. To do this we suppose the contrary and obtain a contradiction. Uniform boundedness would imply by the proof of Theorem 1 , that the net $\left\{P_{\Delta}: \Delta \in \mathscr{D}\right\}$ converges strongly to a projection $P$. Now for each $A \in \mathscr{C}, P_{\Delta}(A)$ commutes with every element $E$ belonging to $\Delta$. Therefore $P(A)$ is in the commutant $\mathscr{E}^{\prime}$ of $\mathscr{E}$. Since also $P(A) \in \mathscr{C}_{1}$ and $\mathscr{E}^{\prime \prime}$ contains no nonzero compact operator, it follows that $P=0$.

However, if $x$ is any unit vector and $\Delta=\left\{E_{1}, E_{2}, \ldots, E_{n}\right\} \in \mathscr{D}$, then

$$
\begin{aligned}
\tau\left[P_{\Delta}(x \otimes x)\right] & =\tau\left[\sum_{i=0}^{n}\left(E_{i+1}-E_{i}\right)(x \otimes x)\left(E_{i+1}-E_{i}\right)\right] \\
& =\sum_{i=0}^{n} \tau\left[\left(E_{i+1}-E_{i}\right) x \otimes\left(E_{i+1}-E_{i}\right) x\right] \\
& =\sum_{i=0}\left\|\left(E_{i+1}-E_{i}\right) x\right\|^{2}=1 .
\end{aligned}
$$

This is true for all $\Delta \in \mathscr{D}$. Since $\tau$ is continuous on $\mathscr{C}_{1}$ this shows that the net $\left\{P_{\Delta}(x \otimes x)\right\}$ does not converge to zero and so contradicts the fact that $P=0$. Therefore the Boolean algebra generated by $\left\{P_{\Delta}: \Delta \in \mathscr{D}\right\}$ is not uniformly bounded.

Another example of this phenomenon arises in a different context in [3]. However in this case the set of projections which generate an unbounded Boolean algebra are only uniformly bounded in norm but are not of norm 1 .

\section{REFERENCES}

1. C. A. Akemann, The dual space of an operator algebra, Trans. Amer. Math. Soc. 126 (1967), 286-302.

2. J. Y. Barry, On the convergence of ordered sets of projections, Proc. Amer. Math. Soc. 5 (1954), 313-314.

3. H. R. Dowson and P. G. Spain, An example in the theory of well-bounded operators, Proc. Amer. Math. Soc. 32 (1972), 205-208.

4. N. Dunford and J. T. Schwartz, Linear operators (Interscience, 1958, 1963, 1971).

5. P. R. Halmos, Measure theory (Van Nostrand, 1950).

\section{KING'S COLLEGE}

LONDON

PRINTED IN GREAT BRITAIN BY ROBERT MIALEHOSE AND CO. ITD PRINTERS TO THE UNIVERSITY OF GLASGOW 